\title{
FORMAS CLINICAS DE LOS ANEURISMAS ARTERIALES INTRACRANEANOS
}

\author{
Alevanidro $H$. Scimoemen \\ Atejandro Schrohidr Otgro
}

Debemos a Dandy ${ }^{1}$ la difusión de los conocimientos de los aneurismas arteriales intracraneanos y la posibilidad de intervenirlos con éxito en muchos casos; este autor describió 108 casos de aneurismas arteriales, algunos de ellos múltiples (15\%). Dandy nunca utilizó la arteriografía debido a los peligros provocados por el Thorotrast, sustancia que se usaba hasta entónces; sobre todo le impresionó la conclusión de Ekstrom y Lindgren (1938), de que en 60\% de los casos inyectados con Thorotrast se producían trombosis cerebrales. Idéntica razón mantenía a nosotros alejados de la angiografía. Pero en 1946, en un viaje a Norte América, conocimos una sustancia que se utilizaba allí y con la cual empezamos a practicar las primeras arteriografías. La inocuidad de la misma está demostrada con la práctica de 400 arteriografías que hemos realizado en el Instituto de Neurologia, sin accidentes.

Hemos utilizado la angiografía en las epilepsias ${ }^{3}$. También la hemos utilizado casi sistematicamente en los tumores cerebrales ${ }^{4}$; la angiografía en los tumores cerebrales no solo permite hacer un diagnóstico de localización sino también, a veces, de la naturaleza del tumor. En los hematomas sub-durales no solo permite diferenciar estos de los intracerebrales y localizarlos con una precisión casi matemática, sino que también es utilísima en casos de localización excepcional ${ }^{5}$. En los quistes hidáti$\cos$ da una imagen bastante característica ${ }^{6}$.

Pero sobre todo nos está siendo utilísima la arteriografía en los casos en que sospechamos de aneurismas arteriales cerebrales, porque ella nos revela que las hemorragias meningeas de los jóvenes, frecuentemente repetidas y a menudo determinando la muerte, no son debidas a meiopragias vasculares como lo enseñaron los autores clásicos, sino que a aneurismas congénitos vasculares; más raramente esos aneurismas son de origen micótico $(4,5 \%$ en la estadística de Dandy) o de origen arterio-esclerótico ( $16,2 \%$ en la estadística de Dandy) en los hombres de edad; casi nunca son de orígen específico.

Aplicando sistematicamente la angiografía a todos los casos que venían a nosotros con el síndrome de hemorragia meningea, con o sin el

Trabajo del Instituto de Neurología de Montevideo (Uruguay). 
síndrome descripto por Dandy (parálisis extrínsecas en los ojos, sobre todo del III par), hemos podido verificar aneurismas arteriales en 30 casos. Como veremos más adelante, hay otras formas clínicas de los aneurismas arteriales fuera de los que producen hemorragias meningeas y determinan parálisis extrínsecas de los ojos.

\section{TECNICA}

Para la arteriografía usamos la técnica transcutánea. Una hora antes de realizarla se inyecta $1 \mathrm{~cm}^{3}$ de atropomorfina; si el enfermo ha tenido ataques epilépticos se le dan $0,10 \mathrm{~g}$ de luminal la noche anterior. Previa a la inyección de Nosylan se hace el test intradérmico y conjuntival. Se coloca el enfermo en decúbito dorsal con la cabeza en hiperextensión. A 3 o $4 \mathrm{~cm}$ por encima de la articulación externo-clavicular y sobre el trayecto de la carótida primitiva palpada previamente se hace anestesia local con novocaína al 1\%, infiltrando los planos superficiales y también los profondos. Luego, con una jeringa de $5 \mathrm{~cm}^{3}$ cargada con suero físiológico y armada con una aguja $n^{\circ} 17$ ó 18 , se punciona la carótida primitiva. Una vez bien canalizada esta, o sea, cuando se tiene la seguridad de estar bien en la luz de la carótida, bombeando suave y repetida vez con el suero de la jeringa, se adapta a la aguja una jeringi de $20 \mathrm{~cm}^{3} \mathrm{con}^{\text {la }}$ interposición, entre jeringa y la aguja, de un tubo de goma con adaptador para la aguja y, en el extremo del tubo adaptado a la jeringa, una llave de doble paso. Se vuelve a verificar la buena canalización de la aguja en la carótida, se cambia entónces la jeringa de suero por otra con Nosylan al 35\% y se inyecta éste en el tiempo de 6 segundos $\left(20 \mathrm{~cm}^{3}\right)$. Se sacan 4 o 6 placas durante la inyección. Se comienza a hacer las radiografías cuando solo quedan $5 \mathrm{~cm}^{3} \mathrm{en}$ la jeringa. Como la circulación cerebral dura unos 6 segundos las placas deben hacerse en cse lapso de tiempo. En el primer segundo se rellenan las arterias; un segundo despues se rellenan las arteriolas y comienzan a llenarse las venas superficiales; en el tercer segundo se llenan las venas profundas; en el quinto se llenan los senos laterales; en el sexto practicamente ya no sa ve relleno de los vasos cerebrales. Despues de terminada la inyección de Nosylan se lava el sistema vascular con $20 \mathrm{~cm}^{3}$ de suero físiológico. Se debe hacer siempre arteriografía lateral y antero-posterior para localizar las lesiones arteriales en su posición endocraneana.

Los enfermos toleran muy bien la arteriografía en la inmensa mayoría de los casos; a menudo los enfermos de policlínica se van directamente a su casa, salvo que se constate una afección grave que obligue a internarlos.

En los primeiros casos de hemorragia meningea en que resolvimos hacer la arteriografía esperábamos que el enfermo mejorara, o sea que desapareciera la rigidez de la nuca, el estado de sommolencia o de coma en que nos llegaba. Pero la triste experiencia de 4 casos de muerte por una recidiva de la hemorragia durante la espera para hacer la arteriografía, nos obligó a cambiar de conducta. Hoy hacemos la arteriografía cualquiera que sea el estado en que llegue el enfermo. Ia experiencia nos ha mostrado que los enfermos no einpeoran con la arteriografía; y que es mas aún, que a veces los enfermos mejoran sin una explicación plausible.

También hemos cambiado de conducta en cuanto al momento de hacer la terapéutica quirúrgica de los aneurismas vasculares. En los primeros casos preparábamos el ennfermo con la compresión diaria progresiva hasta llegar a 30 minutos de compresión digital para hacer la ligadura de la caróticia, ya primitiva, ya de la carótida interna, segun los casos. Hoy, a los enfermos que llegan con sospecha de aneurisma vascular arterial, se les hace la arteriografía de irmediato y también la terapéutica quirúrgica de inmediato en las condiciones que más abajo se verá leyendo los casos que van a continuación. 


\section{FORMAS CLINICAS}

Exponemos algunos casos típicos para la clasificación de las formas clínicas que hemos observado en los aneurismas vasculares cerebrales. Es muy probable que en la medida que nuestra experiencia en los aneurismas arteriales cerebrales se amplie, tengamos que extender el cuadro de las formas clínicas con que se presentan los aneurismas arteriales cerebrales: 1) La forma más frecuente és la de la clásica hemorragia meningea; 2) Otra forma bastante frecuente és la descripta por Dandy, o sea con parálisis de los músculos extrínsecos de los ojos, acompañada de dolores en el territorio del trijémino; 3) Las formas pseudo-tumorales: cefaléa de mayor o menor duración acompañada de atrofia de un nervio óptico y de parálisis de los musculos extrínsecos de los ojos, o cefaléas seguidas de alteraciones psíquicas o embotamiento progresivo sin síntomas focales, ni parálisis oculares; 4) La forma pseudo-encefalítica, en la que el enfermo en plena salud es atacado de cefaléas, fiebre y somnolencia; 5) La forma con epilepsia clínicamente esencial sin síntomas de localización y sin síntomas de hipertensión.

\section{OBSERVACIONES}

\section{Formas de hemorragia meningea}

Caso 1 - F. S., 48 años, casado, argentino, examinado en 25-5-1949. Enmerdad actual - Ayer a mediodia estaba bebiendo alcohol $r$ luego del primer sorbo tuvo una crisis de cefaléa paroxística frontal con mareo y colapso.

Exámen - Al ingreso al Hospital, hora 13, paciente de aspecto sudoroso, pálido, quejandose de fotofobia; presión arterial 14-8, pulso 120 . $\Lambda$ la hora 13,30 sigue con cefaléa, fotofobia, palidez y se halla ligeramente confuso. Al exámen: rigidez de nuca franca, pupilas en miosis regulares, mucosas pálidas, vientre defendido donde se palpa un hígado grande, reflejos osteotendinosos universalmente vivos. A la hora 14 hace un chucho violento con pérdida de materias, inquietud, agitación y mayor confusión mental; signo de Babinsky espontáneo a derecha; rigidez de nuca en tabla; pupilas en miosis; reflejos vivos; presión arterial 21-12; pulso 90. $\Lambda$ la hora 15 el enfermo está casi en coma, con $3 \tau, 5^{\circ} \mathrm{C}$ de temperatura axilar; pulso y presión igual; no existen paresias; alteraciones del ritmo respiratorio sin tipo definido; inquietud; signo de Babinsky bilateral. A la hora 16 el paciente entra en coma franco, con $38^{\circ} \mathrm{C}$ de temperatura. A la hora 20 , coma profundo; síndrome meningeo; respiración profunda, miosis puntiforme. Fl exámen mostró: enfermo en coma profundo, sudoroso; fondo de ojo con papilas normales y arterias con reflejo central algo aumentado sin irregularidades de calibre, ni signos de Gunn; retina sin particularidades; miosis bilateral; al levantarle los párpados se ve una mirada conjugada hacia abajo; no hay rotación cefálica; rasgos simétricos; rigidez de nuca intensa; signo de Babinsky bilateral; pulso 140 regular; presión arterial $18-10 ; 40$ respiraciones por minuto, profundas, esbozando un ritmo de Cheyne-Stokes. Se hace la punción lumbar extrayendo $2 \mathrm{~cm}^{3} \mathrm{de}$ líquido ligeramente hemorrágico. El enfermo sale del coma en 3 dias, con oxígeno permanente y coramina cada 6 horas. Al $5^{9}$ dia hace una congestión pulmonar y entra en coma con hemiplegia derecha. Se le hace arteriografia en coma.

Arteriografía (7-6-1949) - Se hace arteriografía izquierda con placas laterales y antero-posteriores. Se obtiene un relleno vascular bastante bueno que muestra la existencia de un pequeño aneurisma izquierdo por encima y por de- 
lante de la silla turca y los vasos del territorio de la silviana bruscamente amputados en los dos tercios de su trayecto (fig. 1). La angiografía hace pensar que se ha colectado un gran coágulo por debajo del lóbulo temporal. Se resuelve, de acuerdo a este resultado, intervenir con urgencia.

Intervención (7-6-1949) - Se hace un colgajo izquierdo descubriendo el lóbulo temporal. La dura-madre aparece oscura como si por debajo hubiera sangre colectada. Abierta esta parece una hemorragia sub-dural difusa, abundante, que se extiende por sobre todo el cérebro expuesto. Se expone la parte profunda del lóbulo temporal y la arteria silviana aparece envuelta en un coágulo organizado. Se abre en algunas partes la aracnoides, saliendo líquido y sangre. Se cierra la dura dejando una pequeña abertura en la parte más baja del lóbulo temporal. Se cierran los demás planos como de costumbre.

El enfermo fallece a los 8 dias de la intervención. La necropsia mostró la existencia de un aneurisma en el círculo de Willis.

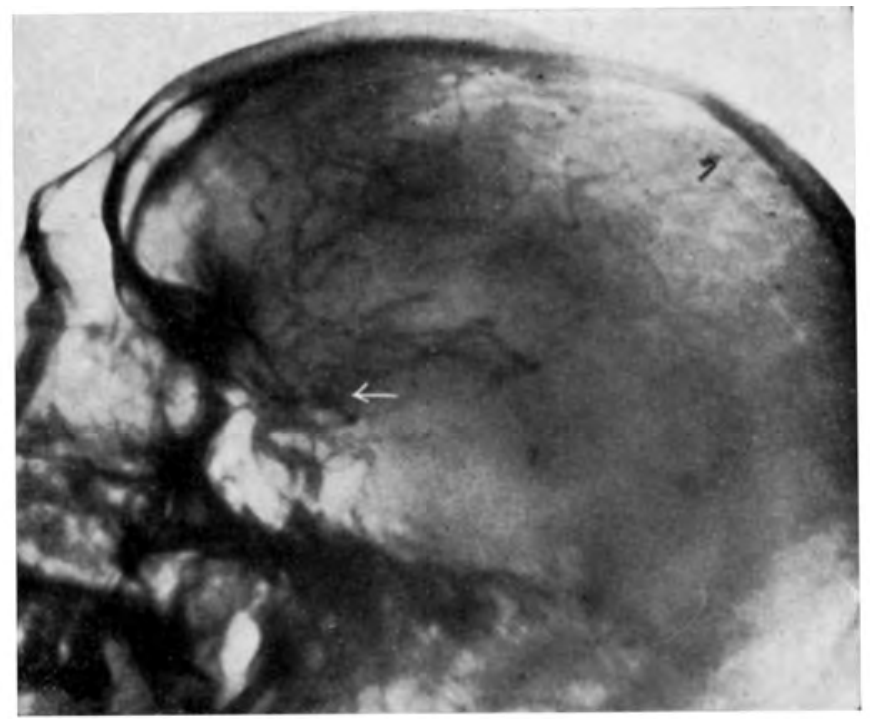

Fig. 1 -.. Caso 1 (F. \$.). P’equeño aneurisma izquierdo por encima y por clelante de la silla turea.

Caso 2 - J. C., 21 años, uruguayo, soltero, vendedor de diarios, ingresado a la Sala Soca del Prof. García Otero, por cefaléas. Enfermedad actual - Hace aproximadamente 48 horas comienza con cefaléa intensa y decaimiento; consulta en la puerta del Hospital donde lo encuentran febril y con un síndrome meningeo; se le hace la punción lumbar saliendo líquido hemorrágico. Antecedentes: Al parecer fué siempre sano. Hace dos años, chancro sifilítico no tratado.

Exámen - Paciente lúcido pero molestado por las cefaléas; estado general bueno; piel y mucosas bien coloreadas; $38^{\circ} \mathrm{C}$ de temperatura axilar; pulso 86; presión arterial 13-8; síndrome meningeo discreto; resto del exámen neurológico y general sin particularidades. Dos dias despues de internado aparece ptosis discreta del párpado derecho con midriasis. Al otro dia aumentan la ptosis palpebral y la midriasis, y se instala una paresia de los movimientos oculares, salvo el de adducción; el enfermo está algo embotado. Al dia siguiente, hemiparesia iz- 
quierda que predomina en la cara y miembro superior. Se decide hacer arteriografía.

Arteriografía - Se hacen placas laterales y antero-posteriores. Se obtiene un bueno relleno vascular, sobre todo en las placas arteriales, que demuestran la existencia de un doble aneurisma del sifón carotídeo derecho, sin que existan desplazamientos de los gruesos troncos vasculares (fig. 2). Como preparación para la ligadura de la carótida se aconseja iniciar la compresión progresiva de la carótida primítiva de ese lado y realizar una arteriografía del otro lado para descartar una malformación semejante controlateral que contraindicara la ligadura. A las 48 horas de realizada la arteriografía el enfermo entra en coma progresivamente.

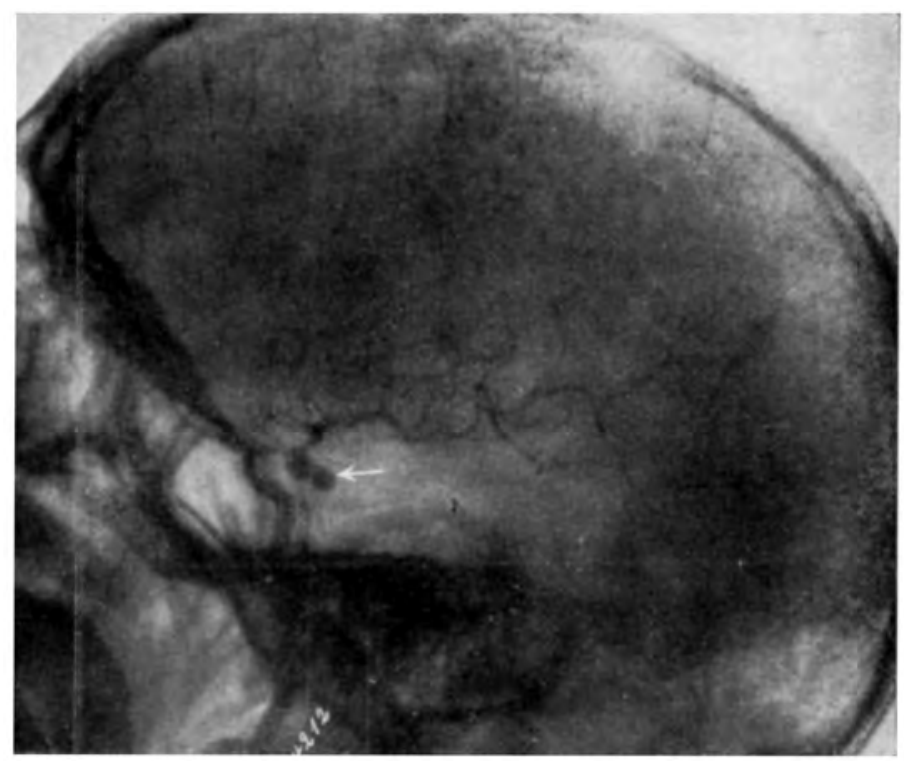

Fig. 2 - Caso 2 (J. C.). Doble aneurisma del sifón carotídeo a la derecha.

Intervención - En la noche, de urgencia, se le hace la ligadura de la carótida primitiva. A la mañana siguiente el enfermo ya ha salido del coma y contesta a algunas preguntas aunque algo embotado. A los dos dias de la ligadura está completamente lúcido, con completo retroceso de la hemiparesia izquierda. Los síntomas oculares no se han modificado.

Evolución - A los 20 dias el paciente es visto nuevamente; ha comenzado a trabajar, se siente bien aunque se queja de una leve cefaléa; la ptosis del párpado ha disminuído y comienza a mover el globo ocular; ha comenzado el tratamiento antisifilítico con mercurio y bismuto.

Este caso tiene de importante, clinicamente, el hecho de que empezó por un cuadro típico de hemorragia meningea (confirmada por la punción lumbar con líquido hemorrágico) y que a las 48 horas aparecieron las parálisis oculares. Es interesante el caso en cuanto demuestra que, aunque tenía sífilis en actividad, ésta no era la causa del síndrome. Quirurgicamente nos enseña que aún estando el paciente en cona y sin preparación previa, la ligadura de la carótida restableció al enfermo. 


\section{Formas con parálisis oculares extrínsecas}

Caso 3 - P. C., 71 años, casado, uruguayo. Enfermedad actual - Saliendo a pasear despues de una abundante comida siente de repente un gran dolor en la nuca y deseos de vomitar; un médico le tomó la presión constatando máxima de 23; fué enseguida para su casa y visto por otro médico, quién le recetó hielo en la cabeza, vitamina $B_{1}$ y cama; a las 24 horas aparece ptosis del párpado derecho y luego cierre total del ojo; cefaléas muy intensas. Antecedentes: A los 60 años fué a consultar médico por unos dolores precordiales y se los trataron como de origen nervioso. A esa edad le constataron presión de 18, yendo en aumento hasta hace dos años en que tenía 27.

Exámen - Enfermo que representa la edad y aún más; memoria algo debil. Ojo izquierdo: bien. Ojo derecho: bordes papilares borrosos. Pupila derecha más grande que la izquierda. La derecha no reacciona ni a la luz ni a la acomodación. El ojo izquierdo conserva los movimientos. El ojo derecho no lo puede elevar y existe paresia interna. Ios reflejos osteotendinosos en los miembros superiores salen débiles de ambos lados; reflejos rotulianos y aquilianos no salen de ambos lados; signo de Babinsky a la izquierda; signo de Oppenheim a la derecha; resto del exámen sin particularidades.

Arteriografía (24-3-1950) - Aneurisma del sifón de la carótida derecha (fig. 3).

A este enfermo no se le hizo terapéutica quirúrgica porque teniendo 71 años era muy peligrosa la ligadura de la carótida, aún con la preparación previa de compresiones cada dia mas prolongadas hasta 30 minutos como se hizo en el caso 8 . También era grave el abordaje directo del aneurisma intracraneano. El enfermo, una ve en la cama y puesto en tratamiento dietético y medicamentoso, tenía 18 de presión. Vivió hasta los 71 años con su aneurisma; un desarreglo en la vida provocó una crisis de hipertensión que produjo la ruptura del ancurisma.

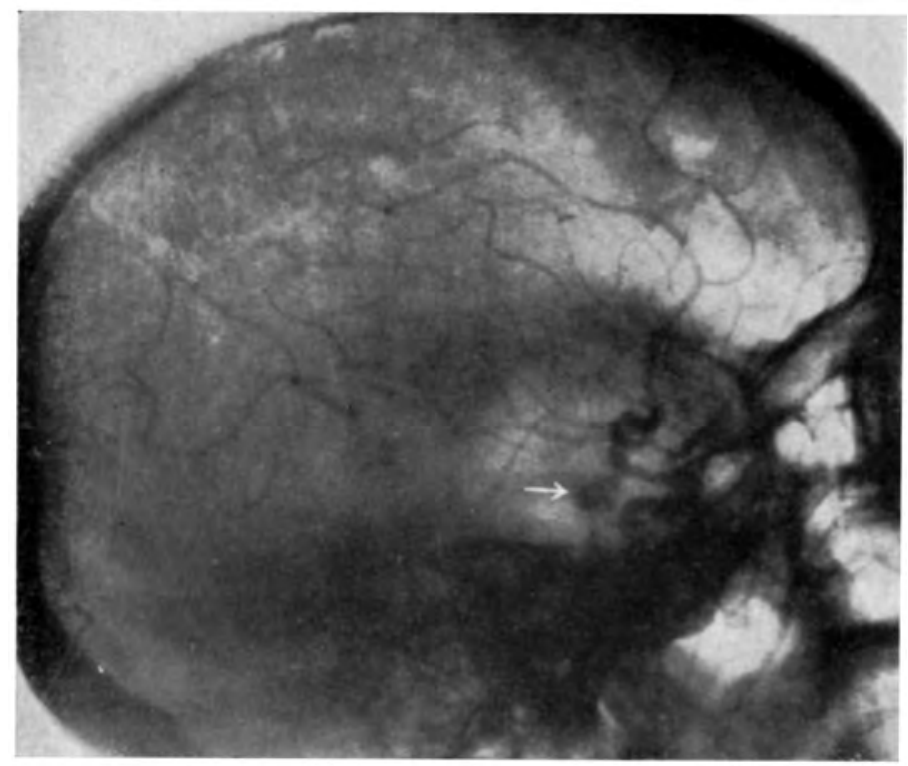

Fig. 3 - Caso 3 (P. C.). Aneurisma del sifón de la carótida derecha. 
CAso 4 - M. R. L. P., 51 años, casada, uruguaya. Enfermedad actual Comienza hace dos meses con cefaléa supraorbitaria izquierda que se irradía a región occipital izquierda, apareciendo en cualquier momento del dia, durando varias horas y se aconpañando, en sus exacerbaciones, de mareos, náuseas y vómitos; desde hace 14 dias, dolor particularmente intenso, apareciendo en horas de la mañana y durando uno o dos minutos; desde hace 8 dias, disminución de la hendidura palpebral izquierda con visión doble; desde hace 6 dias, ptosis palpebral total. Antecedentes: hace 3 años, proceso inflamatorio en la rodilla derecha; desde hace 3 meses está en amenorréa.

Exámen - Buen estado de nutrición y general; mucosas bien coloreadas; temperatura $37^{\circ} \mathrm{C}$. Papila izquierda algo congestiva; pupila derecha $y$ sus reflejos normales; pupila izquierda en midriasis acentuada con abolición de los reflejos; ptosis completa del párpado izquierdo, estando el ojo desviado hacia arriba y afuera; parálisis total del motor ocular comun, extrínseca y intrínseca; reflejo corneano izquierdo disminuído; hipoestesia de rama superior izquierda del trijémino; muy ligera hipoestesia de rama media y sensibilidad normal de la rama inferior; surco naso-geniano izquierdo menos acentuado que el derecho; el estudio de los movimientos de la cara no revela ninguna parálisis; resto del exámen neurológico normal T'onos cardíacos bien golpeados; segundo tono de timbre algo alterado; pulso regular (68). Presión arterial 13-7; la enferma relata que tuvo una vez 18. Exímenes de laboratório - Orina normal. Wassermann en la sangre negativa.

Arteriografía (13-4-1951) - Existe una doble deformación aneurismal franca del sifón carotídeo izquierdo en su porción superior terminal, próxima a su bifurcación, de tamaño mediano $y$ de contornos nítidos. Estos hechos se comprueban tanto en la placa lateral como en la antero-posterior. Existe además en la lateral un discreto levantamiento del grupo silviano de ese lado (fig. 4).

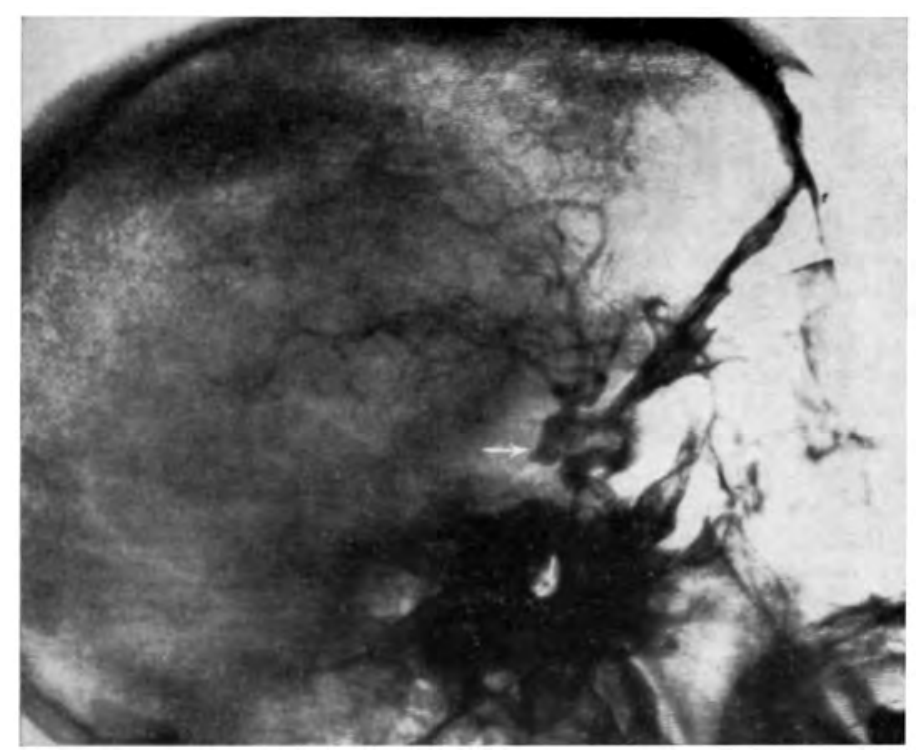

Fig. 4 -. Caso 4 (M. R. L. P.). Doble deformación aneurismal del sifón carotídeo a la izquierdia. 
A las 6 de la mañana siguiente la cefaléa exacerba, irradiandose a región occipital izquierda, durando 30 o 40 minutos, acompañada de sensación de pulsación intracraneana y de vómitos; se practica un minuto de compresión de la carótida primitiva izquierda. A las 16 horas del dia siguiente se repite el mismo episodio de hipertensión, por lo cual resolvemos intervenir de inmediato.

Intervención (13-4-1951) - Bajo anestesia local se realiza la descubierta de la carótida primitiva del lado izquierdo. Se hace la compresión progresiva del vaso en la mesa operatoria durante una hora sin que la enferma acuse la más mínima deficiencia de ese hemisferio, por lo que se practica la ligadura doble del vaso con hilo grueso de seda. La enferma sale en perfectas condiciones de la mesa operatória.

Evolución - En el post-operatorio inmediato se la vigila cada media hora hasta las 8 de la mañana siguiente, hora en que la enferma estaba perfectamente lúcida, habiendo desaparecido su cefaléa y sus vómitos y pidiendo que le dieran de comer. Despues ha seguido muy bien, comienzando a mover su párpado superior izquierdo. Conserva íntegros los movimientos del hemicuerpo derecho y no tiene alteración de la palabra. Alta el 30 de abril de 1951.

Este caso es típico en las manifestaciones clínicas del aneurisma por parálisis oculares, pero en el se agrega hipoestesia de las dos ramas superiores del trijémino. La ligadura precoz hecha lentamente por los fenómenos amenazantes que tuvo la enferma, con desaparición de los síntomas en el post-operatorio demuestran que la operación estaba bien inclicada.

\section{Forma pseudo-encefalítica}

Caso 5 - O. R., 20 años, casada, examinada en el 3-1-1951. Enfermediad actual - El $1^{\circ}$ de Enero tiene fuertes cefaléas que la obligan a guardar cama. Durante los dos dias siguientes aparecen temperatura $\left(38,2^{\circ} \mathrm{C}\right.$ a $\left.38,5^{\circ} \mathrm{C}\right)$, vómitos y somnolencia.

Exámen - Somnolencia, rigidez de nuca, vómitos frecuentes; fondo de ojo normal; el resto del exámen neurológico normal. El exámen cardíaco enseña una enfermedad mitral típica. En el 6 de Enero aparece borramiento del bordo nasal de ambas papilas; sc le hace punción lumbar que demuestra abundantes glóbulos rojos y $0,30 \mathrm{~g}$ de albúmina por mil; se le indica penicilina y estreptomicina. En el 9 de Enero, la somnolencia es más profunda, el edema de la papila es franco, la cefaléa es más intensa a izquierda; hay un reflejo plantar derecho dudoso; llamada y estimulada la enferma contesta bien a las preguntas.

Exámenes de laboratorio - Exámen de orina: densidad $1.020 ; 33,50 \mathrm{~g}$ de uréa por mil; cloruros, vestigios; $0,45 \mathrm{~g}$ de albúmina por mil; células epiteliales, pocos glóbulos de pus, glóbulos rojos, algunos cilindros hialinos granulosos. Uréa en sangre: $0,34 \mathrm{~g}$ por mil. Glucosa en sangre: 1,70 por mil. Eritrosedimentación: $1^{\text {a }}$ hora, $130 \mathrm{~mm}$; $2^{\mathrm{a}}$ hora, $137 \mathrm{~mm}$. Indice de Katz 85. Hemograma: 3.700 .000 glóbulos rojos por $\mathrm{mm}^{3}$; hemoglobina $78 \%$; valor globular 1,05; glóbulos blancos 10.400 por $\mathrm{mm}^{3}$.

Arteriografía (15-1-1951) - Las laterales y antero-posteriores demuestran un levantamiento franco del grupo silviano en la faz arterial y un aneurisma de medianas dimensiones a ese nivel, lejos de la porción inicial de la silviana. Las placas antero-posteriores confirman lo mismo, mostrando un desplazamiento franco del grupo silviano y, hacia adentro y arriba, un aneurisma de las ramas de la silviana. Se hace diagnóstico arteriográfico de hemorragia colectada temporal izquierda a punto de partida de un aneurisma de las ramas de la silviana (fig. 5).

Intervención - Anestesia general con eter. Colgajo parieto-temporal izquierdo , bajo, mostrando la dura tensa y azulada. Abierta esta, el cerebro hace hernia. Se punciona en la parte más saliente con trócar de Cushing, saliendo $10 \mathrm{~cm}^{3} \mathrm{de}$ sangre negra. Se abre el cerebro en ese sitio con una incisión lineal y, cerca de la corteza, aparece un coágulo y se lava la cavidad con suero. Se busca el aneu- 

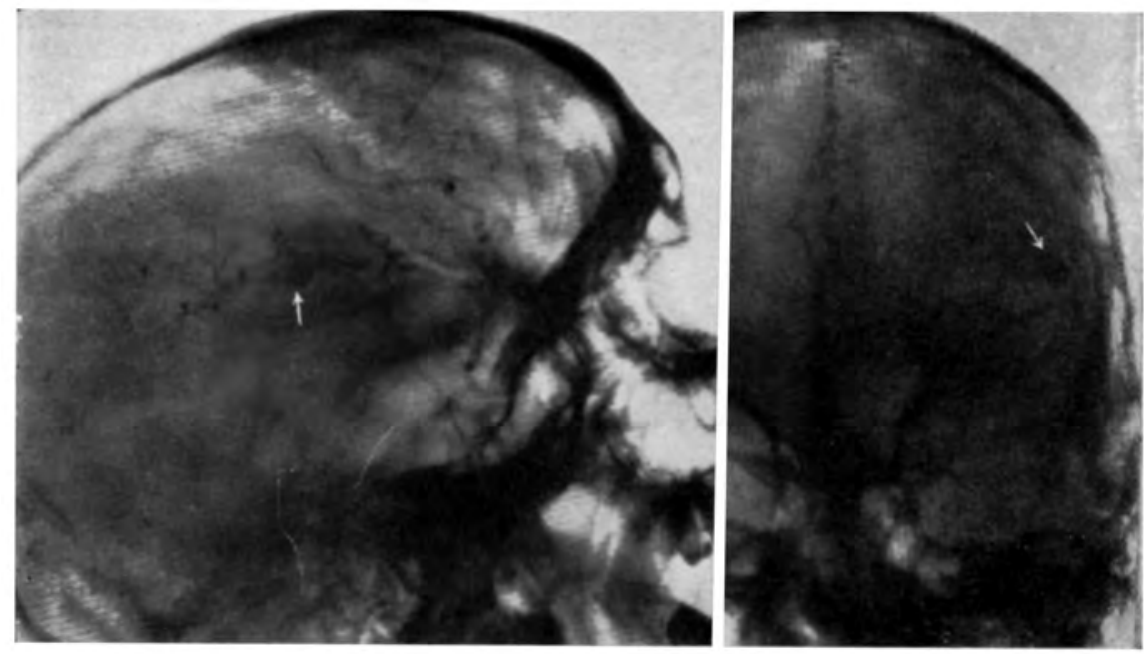

Fig. 5 - Caso 5 (O. R.). I evantaniento $y$ clesplazamiento hacia adentro de las arterias del grupo silviano (colección hemorrágica temporal izquierda); aneurisia lejos de la porción inicial de la silviana.

risma que no se encuentra, dejando "gelfoam" en el fondo de la cavidad $y$ en los puntos que sangran de ella. Al cerrar la dura-madre se deja un pequeño Cushing, pues el cerebro hace hernia.

Post-operatorio - La enferma sale rapiclamente del estado de sueño; desaparecen los vómitos $y$. desciende la temperatura; se empieza a alimentar por la boca dentro de las 24 primeras horas, tolerándolo bien. Alta en perfectas condiciones.

lista enferma empieza su cuadro clínico con cefaléas, vómitos y sommolencia, que se hacen progresivamente más profundos. El exámen del líquido cefalorraquídeo deja ducla sobre la naturaleza de la lesión; podría ser una encefalitis a tipo hemorrágico. La cefaléa predoninante en el lacio izquierdo del cráneo nos guió para el lugar de ta arteriografía. Lin este caso la existencia de la enfermedad mitral y la velocidad de sedimentación hace pensar en la naturaleza micótica del aneurisma causante de la hemorragia.

\section{Formas pseudo-tumorales}

Caso 6 - E. P. de V., 45 años, uruguaya, casada. Enfermedad actual Comienza hace un mes el cuadro, en que el esposo notó, al despertarse, que la enferma tenía una respiración estertorosa, con espuma en la boca y que decía incoherencias al hablarle. Este episodio duró una hora al cabo de la cual recobró la consciencia, quedando con cefaléas fronto-parietales que se irradiaban hacia la nuca; vómitos de alimentos; fiebre de $38^{\circ} \mathrm{C}$ y rigide\% de nuca. $A$ los 15 dias tiene un segundo episodio similar a este que duró un rato. Ha notado ciisminución de la visión desde el primer episodio. Ha tenido sensaciones parestésicas de los 4 niembros pero sin pórdida de fuerzas. No ha tenido trastornos de la micción.

Exámen - Paciente con aspecto febril, se quejando de cefaléas frontales; pulso 66, regular. "En el campo visual, disminución concéntrica a la izquierda $y$ 
un cnsanchamiento de la mancha cicara bilateral. Én la retina del lado derecho, algunos puntos blancos periféricos que parecen de aspecto degenerativo; en cl ojo izquicro, hemorragia retiniana yuxtapapilar situada en la capa de las fibras ópticas (este tipo cle hemorragia se observa frecuentenente en hipcrtensiones intracrancanas bruscas, como por ejemplo en hemorragias meningeas); los bordes de la papila están algo borrosos de ese lado, pero es lógico que esto suceda por la cxtravazación que tiene que haber junto con la hemorragia; en el ojo clerecins no hay edema papilar; las arterias presentan signos de esclerosis de tipo hipertensivo, particulamente en los entrecrumamientos arterio-venosos en doncle hay signos de Gunn bastante marcados" (Dr. Rodríguez Barrios). Pupilas normales; salen los reflejos fotomotor y acomodación-convergencia. No hay rigidez de nuca, jero duele la cabeza en la flexión hacia aclelante. Cono muscular normal en los miembros superiores; fuer\%as disminuídas en la extensión del antebrazo izquierdo; motilidad conservacla; coorclinación normal; los reflejos bicipitales salen bien; el resto de los reflejos osteo-tendinosos débiles. Los reflejos cutáneos abdominales no salen. En los miembros inferiores, hipotonía nuscular; fuerza algo disminuída en la extensión de las piernas; motilidad conservada; coordinación normal; reflejo rotuliano izquierdo muy débil; el derecho no sale; reflejos aquilianos bien; no hay Babinsky; no hay clonus; signo de Oppenhein bilateral. En la posición parada y con los ojos cerrados lit paciente tiene oscilaciones, sobre todo antero-piosteriores; la marcha es lenta con sensación de que las piernas le van a clauclicar; oscila algo hacia ambos laclos.

Arteriografía - Fl angiograma derecho muestra la existencia de un aneurisma pediculado de la comunicante anterior o de la cerebral anterior dereclia (fig. (j). El resto del angiograma es normal. Pàa descartar la existencia de lesiones bilaterales se hace arteriografias del otro ladio, que demuestran un angiograma normal y que el aneurisina no se llena por ese lado.

Intercención - Anestesia general con pentotal-eter y local con novocaína. Colgajo frontal estético derecho. Abierta la duramadre alparece el cerebro alga

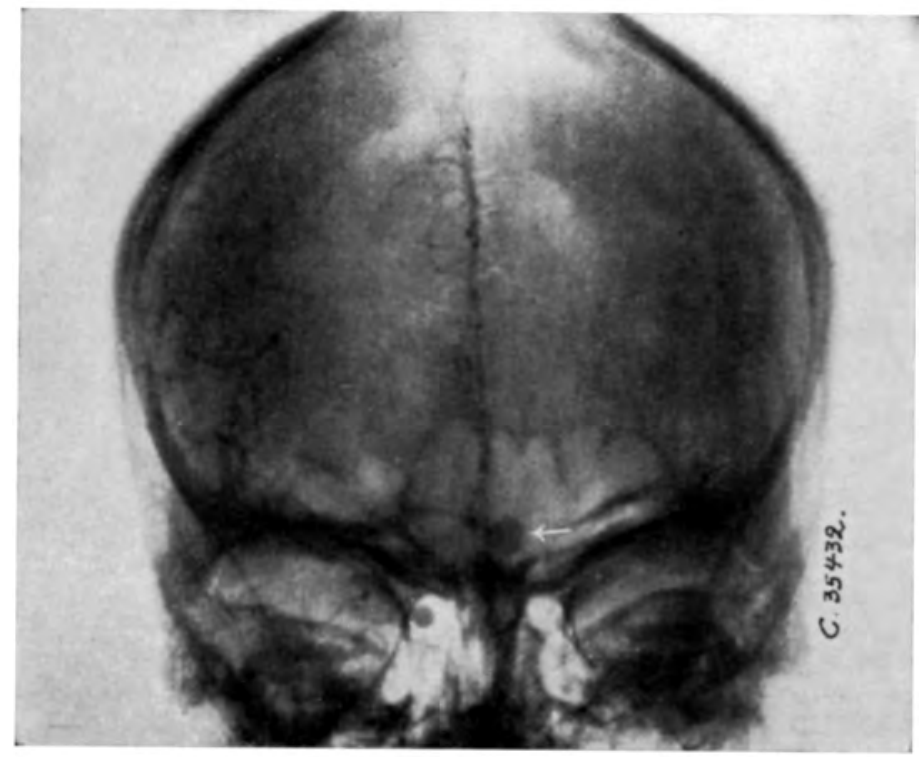

Fig. 6 - Caso 6 ( H. l'. $\mathrm{l}^{r}$.). Aneurisina pediculado de la comunicante anterior o de la cerebral anterior clerecha. 
edematoso y congestivo. El abordaje cle la comunicante se realiza por la linea media, reclinando el lóbulo frontal para arriba y afuera. Se llega así a visualizar la comunicante; en la parte inmediata a ella sangra abundantemente con carácter de sangre arterial. Se cohibe la hemorragia nediante compresión local y la doble compresión arterial en el cueilo. Al pretender abordar el posible sitio del aneurisma vuelve a sangrar prof́usamente $y$ con el mismo carácter. Realizamos la misma maniobra y se cohibe nuevamente. En la imposibilidad de visualizar con toda nitidez el punto de partida de la sangre colocamos en el punto que sangra músculo y "gelfoam". Controlada la hemorragia hacemos el cierre sólo con los planos superficiales sin la parte ósea debido a que el edema del lóbulo frontal impide cerrar el plano clural. Sutura de la piel en dos planos con puntos separados de hilo. Vendaje. Previamente a realizar el colgajo se practicó, a través de un orificio parietal derecho, una punción ventricular con el fin de aliviar la tensión cerebral y salió líquido hemorrágico sin mayor tensión. Durante la intervención se le dieron a la enferma $800 \mathrm{~cm}^{3}$ de sangre.

Post-operatorio - $A$ las 4 horas de operada la paciente había recobrado el conocimiento. El resto del post-operatorio fué sin incidencias. A los 10 días de operada, paciente apirética con menos cefaléas $y$ sin ninguna otra molestia. Fué clada de alta al mes de operada, totalmente recuperadit.

Es de notarse en este caso el inicio del cuadro clínico por ataques convulsivos con pérdida de conocimiento seguida de cefaléas $y$ vómitos $y$ un pulso lento. El fondo de ojo con papilas borrosas pero con la hamorragia retiniana yuxtapapilar hizo sospechar la hemorragia meningea que obligó a la arteriografía que hizo el diagnóstico. Este caso por su inicio pudo ser considerado como un tumor cerebral $y$ el aspecto del fondo de ojo lo podenos ver también en los empujes agudos de los tumores cerebrales.

Caso 7 - J. R., 45 años, viuclo, español, conductor de ómnibus, examinado en el 2t de Marzo de 1950. Énfermedad actual - Desde hace 4 meses uno contpañero de trabajo le nota algunas rarezas y distracciones. La enfermedad actual comienza alrededor del 10 de este mes con cefaléas, vómitos y decaimiento. Pudo volver posteriormente al trabajo pero debió abandonarlo por el decaimiento $y$ las cefaléas. Tiene disminución de fueraas de la mano i\%quierda desde la iniciación de este cuadro.

Excimen - Lengua saburral y húmeda; paciente febril $\left(38,5^{\circ} \mathrm{C}\right)$. Paciente orientado relativamente bien en el tiempo $y$ en el espacio; desganado, ideación lenta, a veces hostil, otras veces contestando con bromas durante el exámen; en cuanto se le deja sa duerme; ligera disartria. Papilas muy congestivas y borrosas, menos en los bordes temporales; campo visual clinicamente normal. Ligera paresia facial izquierda tipo central. Fil paciente saca la lengua con desviación a derechia. En los miembros superiores: fuer\%as y tono normales; la maniobra índice-nari\% izquierda ni derecha no se laacen bien debido al estado psíquico clel enfermo, pero a izquierda impresiona como una ataxia; hiperreflexia a la d:zrecha; a la izquierda los reflejos salen bien. Reflejos cutáneos abdominales débiles a la izquierda. Fn los mimbros inferiores: paresia izquierda; a derecha la motilidad es normal; la prueba talón iqquierdo-rodilla derecha no es normal; reflejos ostectendinos:s se obtienen a izquierda y son muy débiles a derecha; reflejo cutáneo plantar derecho normal; a la izquierda, dudoso. Presión arterial 15-9. Lixamenes de laboratorio - Uréa en sangre 0,33 g por mil. Reacción de Wassermann en sangre negativa. Hemogramı: 4.500 .000 glóbulos rojos por $\mathrm{mm}^{3}$; hemoglobina 96\%; valor globular 1,06; glóbulos blancos 9.700 por mn³ (neutrófilos $77 \%$, eosinófilos $1 \%$; monócitos $4 \%$, linfócitos $18 \%$ ). Tiempo de coagulación 6 minutos. Tiempo de sangrín 2,30 minutos. Electrencefalograma: disminución de voltaje en todo el hemisferio derecho.

Arteriografía - A la derecha $y$ en proyección lateral, doble aneurisma proyectado sobre la silla turca. Fin lit proyección antero-posterior se ve un anell- 
risma cerca de la silla turca $y$ otro en el trayecto de la silviana, vecino al punto en que esta pasa a la convexidad cerebral (fig. $\tau$ ).
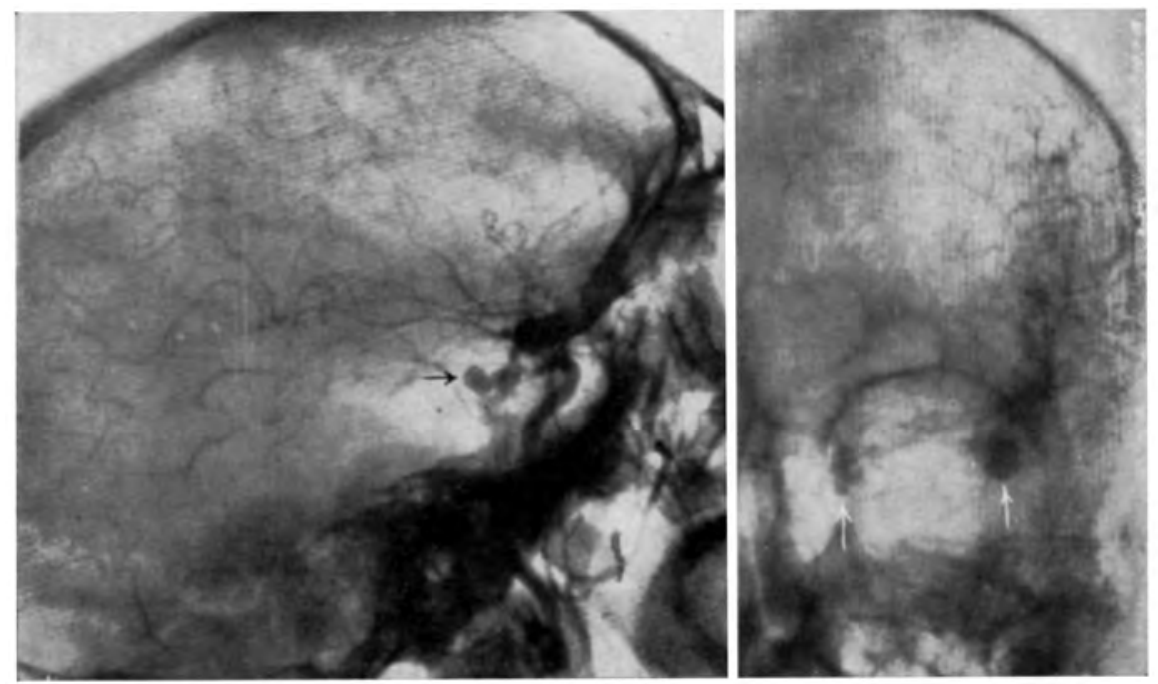

Fig. 7 - Caso 7 (J. R.). En projección lateral, doble aneurisma provectado sobre la silia turca. En projección antero-posterior, un aneurisma cerca de la silla turca y otro en el trayecto de lia silviana, vecino al punto en que esta pasa a la convexidad cerebral.

Operación (21-1-1950) - Anstesia general y local con novocaína a $1 \%$ Colgajo para exponer la fosa temporal y visualizar el aneurisma que estí junto a la silla turca. Previamente se le había descubierto la carótida interna derecha y pasado una ligadura que se dejó suelta para que, en caso de que uno de los aneurismas se rompiera en la exploración intracraneana, pudiera ser ligada rapidamente. Se explora cuidadosanente el polo temporal siguiendo el valle de la silviana de afuera a adentro, no encontrando aneurisma ni signo de ruptura em todo ese trayecto. luego se explora la clinoide onterior derecha $y$ el nervio óptico de ese lado, maniobras que se realizan con el separador de iluminación. Se produce una hemorragia inportante, que se repite cada vez que se intenta ver el aneurisma. Se tiene la sensación de que ella procede del aneurisma que la arteriografía señala en ese sitio. En la imposibilidad de poner un clip al pedículo de ese aneurisma que no se pueds visualian, se resuelve cerrar. Se cierra también la hericla del cuello sin hacer la ligadura de la carótida interna.

Post-operatorio -- El enfermo ha tolerado perfectamente la intervención.

En el 14 de Septiembre de 1950 se le hizo la arteriografía del lado izquierdo, que demostró no haber aneurisma en ese lado. Se le hace entónces la ligaclura de la carótida prinitivi derechil, que fué perfectamente tolerada. Lin el 16 de Septiembre de 1950, el estado del eniermo es 11 uy bueno; no presenta ni la más ligera hemiparesia. P'osteriomente volvió a su trabajo de chauffeur de ómnibus.

El inicio de este caso con sus alteracioncs psíquicas progrcsivas y a las cuales se le agregan cefaléas, rómitos y papilas borrosas, hace pensar en un tumor o absceso. I a disminución de voltaje de toclo un hemisferio volvió el diagnóstico para hemorragia meningea que la arteriografía confirmó por la presencia de dos 
aneurismas de la silviana. Ia terapéutica ideal no fuć posible, o sea poner un clip en el pedículo del aneurisma que sangraba. Pero la ligadura de la carótida demostró que esta también es eficaz.

Caso 8 - M. G. de P., 65 años, viuda, uruguaya, examinada en el 2.4 de Septiembre del 1948. Hinfermedad actül - Hace 2 años comenzó a perder la visión del ojo izquierdo hasta perderla totalmente 5 meses despues. Poco tiempo despues comienza a notar la ptosis del párpado superior de ese lado, que se fué haciendo progresiva $x$ completa poco despues de su ceguera. Nunca tuvo diplopia ni dolores. Desde hace 5 neses sensaciones parestésicas al nivel de las ramas media y superior del trijémino; también tiene obstrucción nasal del lado izquierdo a partir de la misma fecha. Desde hace algunos meses acusa flojedad en los miembros inferiores. Antecedentes: reacción de Wassermann en la sangre repetidamente negativa; hipertensión arterial desde hace algún ticmpo (Mx. 18); poliuria con polaquiuria nocturna desde hace algún tiempo.

Exámen - Paciente apirética en regular estado de nutrición; palidè de piel y mucosas; arco senil marcado. Psiquismo y lenguaje de acuerdo a la edad. Atrofia óptica a izquierda; papila derecha normal. $\Delta$ nisocoria: ojo izquierdo en midriasis, no reaccionando a la luz y con abolición del reflejo consensual; en el ojo derecho, reflejo fotomotor y consensual normales. I’tosis palpebral izquierda. Globo ocular izquierdo desviado hacia afuera, casi inmóvil, aunque insinúa paqueños movimientos de lateralidad. Abolición del reflejo corneano izquierdo; muy discreta hipoestesia a todas las formas de la sensibilidad en el territorio del oftálmico izquierdo. Ligera hipoacusia bilateral; pruebas calóricas normales, salvo que el ojo izquierdo, que está fijado para los movimientos, reacciona my poco. Miembros superiores adelgazados, con hipotonía; los reflejos bicipitales salen bien en ambos lados; Ios otros reflejos nuy débiles. Reflejos cutáneos abdominales no son obtenidos (paredes nuy flácidas). En los mienıbros inferiores: masas musculares disminuídas globalmente; hipotonía marcada bilateral; fuerzas algo disminuídas globalmente; movimientos activos normales; reflejos tendinosos algo débiles; reflejos cutáneos plantares en flexión; hipopalestesia bilateral. Marcha dificultosa, sólo posible si la enferma se apoya en otra persona; discreta tendencia al taconéo. 'Taquicardia; esbozo de ritno a 3 tiempos en la punta; presión arterial 14,5-8,5. Electrocardiograma: Taquicardia (ventricular) sinusal de 103 por minuto; espacio $\mathrm{PR} 0,20$; preponderancia ventricular izquierda; onda $\mathrm{T}$ positiva en las tres derivaciones. Eximenes de laboritorio - Orina normal. Uréa en sangre $0,24 \mathrm{~g}$ por mil. Reacción de Wassermamn en la sangre negativa. Líquido cefalorraquideo incoloro; Pandy debilmente positivo; Nonne dudoso; albúmina $0,30 \quad z$ por mil; citología 2,7 por $\mathrm{mm}^{3}$. Hemograma: glóbulos rojos 3.600 .000 por $\mathrm{mm}^{3}$; hemoglobina $75 \%$; valor globular 1,04 ; glóbulos blancos 7.400 por $\mathrm{mm}^{3}$. Estudio radiológico: Silla turea de diámetros agrandados con las clinoides posteriores poco visibles. Una placa enfocando los agujeros ópticos muestra poco nitidez del contorno inferior del agujaro óptico del lado izquierdo. La hendidura esfenoidal de ese lado también se ve borrosa. Cronaximetria de los músculss maseteros: Masetero derecho normal; en el masetero izquierdo no fuś obtenida respuesta con la intensidad máxima tolerada por la enferuna.

Arteriografia -- Gran aneurisma que asienta en el sifón carotídeo izquierdo y que dilata mucho lì silla turca (fig. 8).

Fn el 21 de Enero de 1949 se inicia la compresión manual y gradual de la carótida primitiva izquierda en el cuello, que la enferma tolera muy bien; $\mathbf{s}$ z llega hasta 30 minutos de compresión.

Intervención (23-2-1949) - Anestesia local con novocaína. Incisión horizontal a la altura del cartílago cricoides. Descubierta de la carótida primitiva. Se pasa un trozo de fascia lata extraído previamente de la misma enferma. Se cose con dos puntos de seda fina. Sobre la tira de fascia lata se hace una ligadura de seda gruesa. Se espera una hora antes de cerrar la inerida. 


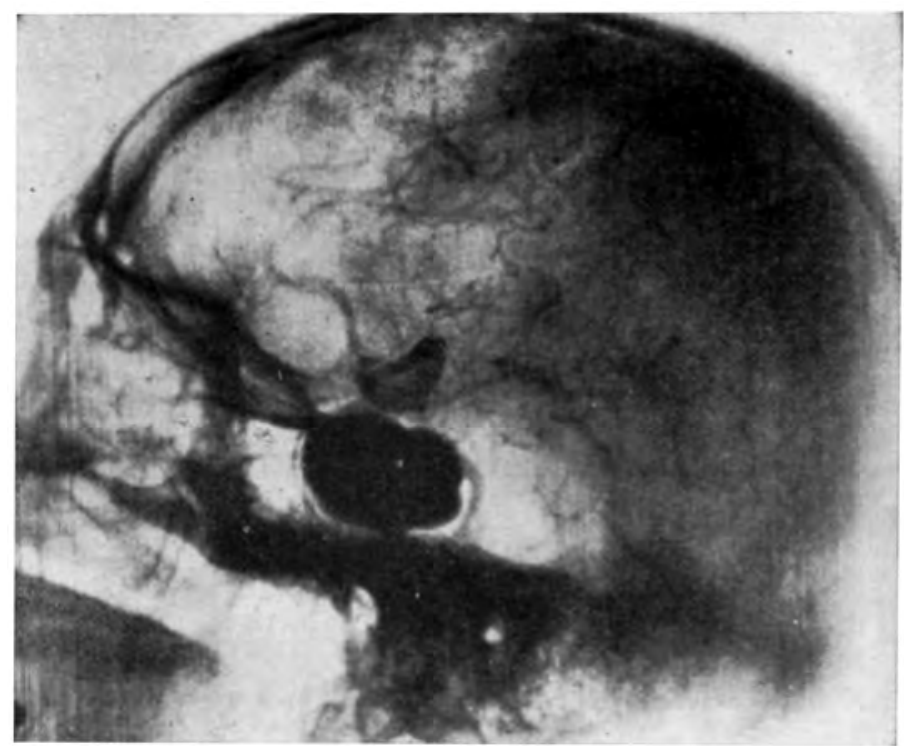

Fig. 8 - Caso 8 (M. G. ए'.). Gran ancurisma en el sifón carotídeo izquierdo y que dilata mucho la silla turoa.

Post-operatorio excelente. La paciente ha pasado perfectamente; no hay modificaciones neurológicas en los nniembros derechos; el pulso temporal y facial se palpa perfectamenie a derecha y no se siente a iqquierda. Alta en el 23 de Abril de 1949: poco han cambiado los sintoras oculares y los trastornos sensitivos subjetivos y objetivos de la sensibilidad de la cara.

La pérdida progresiva de la visión, seguida de atrofia del nervio óptico con parálisis de los nervios extrínsecos y hipoestesia de la primera rama del trijémino y con una dilatación y erosión de la silla turca, hizo pensar en un tumor benigno por laráa evolución. La arteriografía denostró que era un enorme aneurisma que hemos detenido en sua evolución con la ligadura de la carótida primitiva mediante previa preparación por compresión progresiva.

\section{Forma de ep:lepsia clínicamente esencial}

Caso 9 - H. V., 34 años, soltero, examinado en Agosto de 1951. Enfermedad actual - Desde hace 2 años ataques de epilepsia que comienzan con pérdida brusca de conocimiento $y$ en las que tuerce los ojos y se muerde los labios; no recuerda nada del ataqus, ni de lo que lo preceds. Ha tenido alrededor de 20 ataques, los más de dia; de noche sólo ha tenido 3 ataques.

Exámen - Sólo tiene el reflejo plantar derecho en débil flexión y el izquierdo en viva flexión. Todos los exámenes serológicos negativos. Ellectrencefalograma: actividad $\delta$ en la región temporal derecha $y$ actividad epileptiforme de diversos tipos; durante el sueño las alteraciones se hacen todavía más evidentes y a veces se bilateralizan sincrónicamente, lo cual lace pensar que la lesión es profunda en aquallas regiones.

Arteriografía -.- Aneurisra en el trayecto de la silviana en la porción en que comienza recorrido havia la corteza cerebral, a 4 o $5 \mathrm{~cm}$ de su nacimiento (fig. 9). 

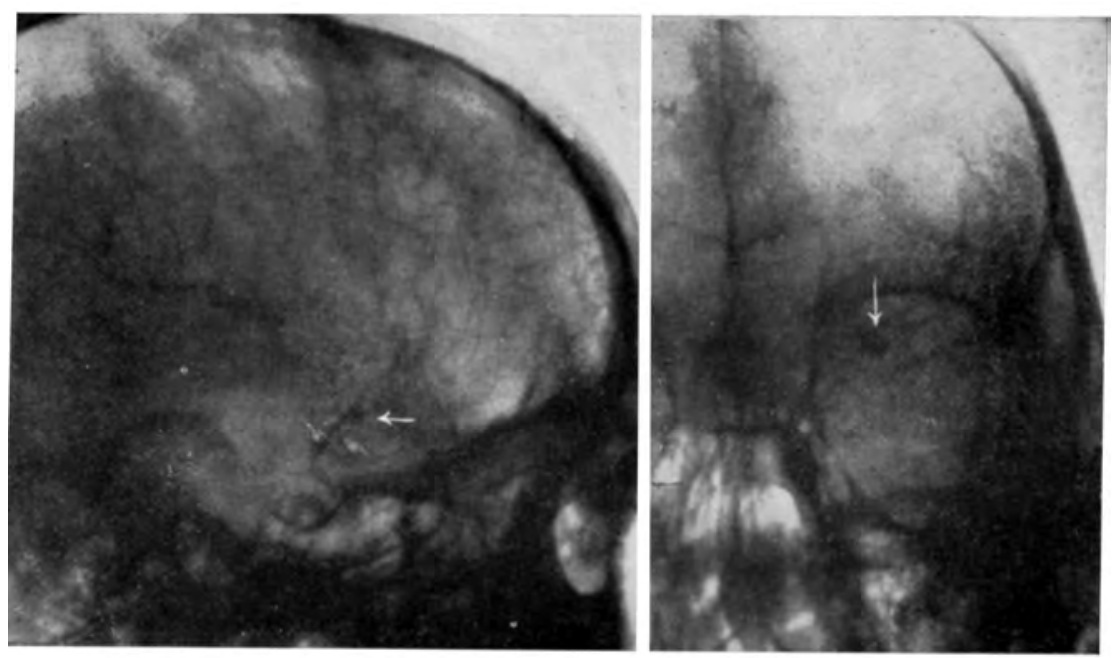

Fig. 9 - Caso 9 (H. V.). Ancurisma en el travecto de la silviana derecha en la porción en que comienza recorrido hacia Ja cortesa cerebral, a 4 o $5 \mathrm{~cm}$ de su nacimiento.

Intervención -- Colgajo temporal derecho para exponer cl lóbulo temporal, apareciendo la silviana muy levantada como en la arteriografía. Se punciona con trócar en la dirección del polo temporal saliendo 10 a $15 \mathrm{~cm}^{3}$ de líquido con glóbulos rojos (cste liqquido en el exámen dió $0,80 \mathrm{~g}$ de albúmina por litro). Se abre el polo temporal con una incisión con bisturí eléctrico y con el aspirador se va abriendo la materia cerebral hasta caer en una pequeña cavidad a paredes lisas y en cuya porción profunda aparece el aneurisma del tamaño de un garbanzo chico. El aneurisma es sesil a la arteria silviana, sangrando continuamente. En la imposibilidad de ligar el aneurisma se coloca un tro:o de músculo temporal de $3 \mathrm{~cm}$ de largo $y 1$ de ancho y, encima del músaulo, dos trozos de "gelfoam". se hace hemostasis cuidadosa, se cierran los diversos planos dejando en la duramadre, en su parte inferior, una abertura de $3 \mathrm{at} \mathrm{cm}$ de largo.

Post-operatorio - Alta 10 dias despues, no habiendo ninguna reincidencia. Se le prescriben Theantoin ( 2 cápsulas al día).

\section{CONSIDERACIONES FINALES}

Insistimos en que la clasificación que hemos presentado de las formas clínicas de los aneurismas arteriales intracraneanos es una nota previa a la clasificación definitiva de las mismas.

Los casos descriptos van precedidos en su mayoría de cefaléas antes de aparecer los otros síntomas. Por eso creemos que debe pensarse en la posibilidad de un aneurisma arterial intracraneano frente a todo caso con cefaléas a localización sobre todo retro-orbitaria y frontal unilateral. Sobre todo sí en la radiografía de cráneo y en el fondo de ojo no encon- 
tramos causa que explique esas cefaléas. La arteriografía delucidará el caso.

\section{RESUMEN}

Los autores exponen las modalidades clínicas que presentan los aneurismas arteriales intracraneanos, baseando su clasificación en experiencia propia çue es de 30 casos, y exponen 9 casos típicos que describen clínica y artericgráficamente, relatando el método quirúrgico utilizado. El diagnóstico del aneurisma arterial y la localización del mismo se hace siempre de manera precisa por la arteriografía, método que tiene un mínimo de riezgo (los autores han hecho 400 arteriografías sin accidente grave).

Los autores consideran la clasificación propucsta como previa que, a medida que se amplie la experiencia, habrá que modificar o completar. La clasificación empleada es la que va a continuación: 1) formas de hemorragias meningeas; 2) formas con parálisis de los músculos oculares extrínsecos; 3) formas pseudo-tumorales; 4) formas pseudo-encefalíticas; 5) formas con epilepsia clínicamente esencial.

\section{SUMMARY}

The authors explain the clinical aspecis presented by the arterial intracranial aneurisms. They base their classification in their own experience which is of 30 aneurisms and present 9 typical cases which they describe clinically and arteriographically, and the surgical method used. The diagnostic of the arterial aneurismas and its localization is always accurately obtained by the arieriography (the authors have made 400 arteriographies without a serious accident).

They consider the classification that follows as previous and susceptible of being modified or enlarged according to experience: 1) form with meningeal hemorrhages; 2) form with paralysis of the extrinsic muscle of the eyes; 3) pseudo-tumoral form; 4) pseudo-encephalitic form; 5) form with epilepsy, clinically essential.

\section{BIDLIOGRAFIA}

1. Danciy, W. -- Intracranial Arterial Aneurysms, 1944.

2. Rogers, $\mathbf{L}$. - Carotid ligation for intracrinial aneurysms. Report of a case studied by electroencephalography. 13rit. J. Surg., 33:369-311, 1944 .

3. Schroeder, A. H., Arana, R., Fuster, 13., Gomensoro, J. I3. y Schroeder Otero, A. M. - Contribución al tratamiento quirúrgico de la epilepsia. An. Fac. Med. de Montevideo, 35:321-364, 1950.

4. Schroeder, $\Lambda$. H. - I a angiografía en los tumores cerebrales. Actas de! Congreso Internacional de Cirugía, Bu’nos Aires, 1950 (en prensa).

5. Schroeder, A. H., A rana, R. y San Julian, J. - Hematoma subdural optoquiasmático. An. Fac. Med. de Montevideo, 36:17-20, 1951.

6. Schroeder, A. H. - Quiste hidático cerebral. IV Congrreso Internacional Sudamericano de Neurccirugía, P'ôrto Nagre, 1951 (en prensa). 